\title{
METODOLOGIAS ATIVAS NA ARTE DE ENSINAR
}

\author{
BLUMENAU/SC MAIO/2018
}

\author{
SIMONE ERBS DA COSTA - UNIASSELVI - simoneerbsdacosta@gmail.com \\ Danice Betania de Almeida - UNIASSELVI - danicebetania@gmail.com \\ Greisse Moser Badalotti - UNIASSELVI - greisse.moser@uniasselvi.com.br
}

\author{
Tipo: Relato de Experiência Inovadora (EI) \\ Categoria: Métodos e Tecnologias \\ Setor Educacional: EDUCAÇÃO SUPERIOR
}

\begin{abstract}
RESUMO
A aprendizagem dos discentes é um dos grandes desafios da docência, buscando que o aluno tenha uma participação maior no processo, de forma interativa e ativa. Dessa forma, foi realizada uma pesquisa e avaliação na educação superior, tendo como objetivo mostrar relatos de práticas realizadas em disciplinas de computação, utilizando metodologias ativas e dispositivos móveis no processo de ensino aprendizagem. Foi utilizado o método de pesquisa quantitativa, aplicado em uma amostragem de 74 sujeitos participantes de cursos de graduação de computação. Cabe destacar, que a proponente vem aplicando esses instrumentos em suas práticas em sala de aula com alunos do ensino superior desde o primeiro semestre do ano 2015. Assim, foi possível concluir que os instrumentos abordados na pesquisa tiveram impactos positivos, pode-se citar em primeiro lugar um aprofundamento dos alunos na aula, constatando-se uma participação mais ativa nas atividades em sala de aula ao se fazer uso de metodologias ativas, quando comparadas as metodologias tradicionais. Também foi possível perceber que os alunos ficam mais motivados e estimulados pelo tema estudado. Ressaltasse que foi observado um impacto considerável quanto a participação dos alunos, quando comparadas as duas metodologias. Os alunos passam a comentar suas experiências frente à turma, tecendo comentários pertinentes ao tema estudado em aula, acarretando em uma maior produtividade com as dinâmicas utilizadas em sala de aula. Dessa forma, foi possível perceber um entendimento do pensamento computacional mais sagaz ao se comparar com as metodologias aplicadas de forma tradicional. Pois, ao se fazer uso das novas metodologias os alunos buscam o aprendizado de cada item, por si só, refletindo com as dinâmicas aplicadas. Por fim, foi possível verificar que as metodologias ativas auxiliam no processo de ensino-aprendizagem.
\end{abstract}

Palavras-chave: Metodologia Ativa. Aprendizagem. Computação. Dispositivos móveis. 


\section{Introdução}

A aprendizagem dos discentes é um dos grandes desafios da docência, buscando que o aluno tenha uma participação maior no processo, de forma interativa e ativa. Nesse cenário, o papel do docente é alterado, "[...] daquele que ensina, ele passa a ser aquele que faz aprender e que também aprende." (DELPHINO, 2015, p. 30). O aluno passar a ter um papel ativo ao invés do papel do aluno passivo, passando a ter uma voz mais ativa nas atividades em sala de aula.

Nesse contexto, as tecnologias educacionais são aliadas interessantes a serem utilizados em sala de aula, auxiliando no acompanhamento individual dos alunos e possibilitando a verificação das possíveis fragilidades perante as atividades que são realizadas (LUCIO, 2015, p. 24). Nessas atividades, as tecnologias digitais acompanham os alunos em sala de aula com ou sem a permissão dos professores, os alunos jogam, assistem filmes, conversam e interagem nas redes sociais.

Essa é a realidade que se tem em sala de aula bem como que os dispositivos móveis estão incorporados no cotidiano dos alunos. Nesse contexto, pode-se tirar proveito desses fatores com o conceito de aprendizagem móvel. A aprendizagem móvel também conhecida como $m$-learning, os alunos fazem uso dos seus próprios dispositivos móveis como forma de diversificar as atividades em sala de aula e ser uma alternativa a ser explorada à falta de infraestrutura tecnológica.

Essa pesquisa tem como objetivo mostrar relatos de práticas realizadas em disciplinas de computação, utilizando as metodologias ativas para disciplinas da grade curricular de computação. Nessas disciplinas foram utilizadas metodologias ativas e dispositivos móveis no processo de ensino aprendizagem, bem como a proponente vem aplicando esses instrumentos em suas práticas em sala de aula com alunos desde o primeiro semestre do ano 2015. Cabe destacar, que o presente estudo foi aplicado com uma amostragem de 74 sujeitos participantes de cursos de graduação de computação, em instituições localizadas nas cidades de Blumenau e Indaial, Santa Catarina (SC).

\section{Metodologias ativas de aprendizagem}

Para melhor entendimento esta seção foi estruturada inicialmente com a contextualização de metodologias ativas e apresenta algumas dessas metodologias que vem sendo utilizadas pela proponente e por seus pares em sala de aula e com três subseções. A subseção 2.1 traz ferramentas aplicadas nessas metodologias ativas; a subseção 2.2 traz um breve resumo dos métodos e materiais utilizados na presente pesquisa; e por fim, a subseção 2.3 apresenta a análise e discussão da avaliação realizada. 
As abordagens tradicionais caracterizam-se pela transmissão direta, sendo 0 conhecimento transmitido de forma estruturado e dialogado. Enquanto as abordagens de ensino aprendizagem inovadoras estão centradas no aluno, e com ênfase no desenvolvimento de habilidades que visam aprender-a-aprender (OECD, 2014). Essas abordagens de metodologias ativas ou significativas fazem parte das abordagens inovadoras. Barbosa e de Moura (2013, p. 55, destaque nosso) colocam que quando existe interação do aluno com o conteúdo como: ouvir, falar, perguntar discutir fazer e ensinar, estimula a construção do conhecimento. Diferentemente de quando não existe interação, sendo entregue de forma formatada ao aluno pelo professor.

O professor passa a atuar não somente como a única fonte de informação e conhecimento, mas sim como um facilitador, supervisor, orientador do processo de ensino aprendizagem. Portanto, os princípios das metodologias ativas basicamente dizem respeito as atividades realizadas pelos alunos que foram destacadas na citação de Barbosa e de Moura (2013, p. 55), de ouvir, falar, perguntar, discutir, fazer e ensinar.

Essas atividades são os norteadores do ensino na aprendizagem ativa, colocando o aluno ativamente no processo de ensino aprendizagem, ao invés do processo expositivo dialogado. Santos e Passos (2016) complementam que as metodologias ativas possibilitam uma participar de forma ativa, contribuir, bem como passam a ter mais autonomia no processo de ensino aprendizagem.

Dessa forma, é necessário fazer uso de estratégias que possibilitam o aluno pensar, ou seja, que ativem o aluno na construção do seu próprio conhecimento. Algumas dessas estratégias dizem respeito a utilização de hardware e software como instrumentos de aprendizagem; a disponibilizar material de apoio como: listas interativas, vídeos, videoaulas, dicas de filmes, tutoriais, e-books. Instrumentos de aprendizagem que estejam baseadas em Tecnologias da Informação e Comunicação (TICs) e aproveitem os próprios dispositivos dos alunos (m-learning).

Essa ideia de os alunos trazerem seus próprios dispositivos móveis é conhecida como Bring Your Own Device (BYOD), sendo conhecida também por Bring Your Own Technology (BYOT), possibilitando funcionários de levarem seus próprios dispositivos móveis (notebooks, tablets, smartphones) para o espaço de trabalho, utilizando-os os próprios aplicativos e informações da empresa. Ao se fazer um paralelo com a escola funciona da mesma forma, sendo solicitado que discentes (alunos) e docentes (professores) que tragam seus próprios dispositivos móveis para que eles possam ser utilizados em sala de aula (PORVIR, 2015, p. 1). 
O objetivo de utilizar esses instrumentos é guiar o aluno, criar um repositório on-line de forma colaborativa, acessível aos alunos e professores que queiram contribuir; fazer uso de diferentes abordagens, como a construção de mapas conceituais (ou mentais), aprendizagem baseada em problemas, aprendizagem baseada em projetos, trabalhos em equipe, estudos de casos, sala de aula invertida, gamificação. Atividades essas que exigem o pensar do aluno.

Educause (2012) coloca a sala de aula invertida como uma inversão da forma em que as atividades são realizadas durante o processo de ensino aprendizagem, dentro e fora da sala de aula, possibilitando utilizar o tempo presencial da sala de aula com atividades práticas com os alunos junto a seus colegas e com o apoio do professor. O conteúdo teórico e expositivo é explorado tanto em sessões à distância como em sala de aula de forma interativa. O método basicamente introduz os conteúdos a serem abordados em sala de aula por meio de atividades que são realizadas antes do momento de sala aula (ALMEIDA, 2017).

A gamificação é uma opção aos modelos tradicionais, uma alternativa para que as pessoas tenham comportamentos mais favoráveis para resolver problemas em outros contextos como de educação, aplicações ou áreas, utilizando elementos e técnicas vistos em jogos. Christianini, De Grande e Américo (2016) colocam que este conceito de gamificação é oriundo da área de design de jogos. Nesses ambientes são estudados os jogos nos aspectos de desenvolvimento, regras, estética, interatividade, narrativa e interface (CHRISTIANINI; DE GRANDE; AMÉRICO, 2016).

Ao utilizar a gamificação e jogos no processo de ensino pode-se destacar a motivação dos alunos, tornando as atividades mais agradáveis ao invés de tediosas ou repetitivas. Klafke (2015, p. 48) complementa que a gamificação “[...] desenvolve o aprendizado por etapas gradualmente mais difíceis, estimula a formação emocional e intelectual, dentre outros. ". Dessa forma, pode-se dizer que o foco está na pessoa do aluno como jogador, avaliando-se a interação dele dentro do sistema que pode ser observada por meio de indicadores, possibilitando rastrear e medir o aprendizado dos alunos.

Relacionado a Aprendizagem Baseada em Problemas (ABProb), o meio para ensinar e aprender é por meio de problemas. Nesta estratégia as atividades podem variam dependendo da forma do ensino e conforme os objetivos que desejasse alcançar (BARBOSA; MOURA, 2013, p. 58). De forma suscinta a etapa que inicial da ABProb é o entendimento e esclarecimento dos problemas para compreender o todo. A segunda etapa diz respeito a geração de ideias a partir do conhecimento atual. A terceira etapa refere-se a análise para que o problema seja dividido em partes, enquanto, a quarta 
etapa diz respeito às questões que guiem a pesquisa e a definição do problema a ser resolvido. A quinta etapa refere-se aos objetivos da aprendizagem; a sexta etapa envolve tanto o estudo individual quanto em equipe, tendo o registro das atividades realizadas. A síntese e avaliação é realizada na sétima etapa, bem como os resultados obtidos. Por fim, na oitava etapa o trabalho realizado é apresentado ao grupo (ARAÚJO, 2011).

Diferentemente da AbProb, na Aprendizagem Baseada em Projetos (ABProj), o meio de aprender e ensinar é por meio de projetos. Krajcik e Blumenfeld (2006, p. 318) colocam que a ABProj "permite aos alunos investigar, questionar, propor hipóteses e explicações, discutir suas ideias, desafiar a de outros e experimentar novas". Dessa forma, os alunos conseguem aprender a partir de um problema real, de conhecer o todo e de suas especificidades a nível de requisitos, ou seja, das funcionalidades determinadas pelo cliente.

\subsection{Ferramentas}

Mentimeter é um recurso digital para realizar interações tanto para grandes grupos quanto pequenos em tempo real e de forma interativa, tornando visível para todos. Essas interações podem ser realizadas em forma de quis, nuvem de palavras, coleta de perguntas e enquetes. Para criar essas interações é suficiente criar uma conta plataforma, escolher o tipo de interação deseja e elaborar a mesma; e para que os participantes tenham acesso ele deverá acessar um link para realizar a votação ou entrar no site www.menti.com e colocar o código de acesso às perguntas.

Caso o Mentimeter esteja sendo projetado, o resultado fica visível para todos em tempo real (INFOGEEKIE, 2017). Cabe ressaltar, que esses recursos podem ser utilizados pelos professores em suas interações com os alunos de forma interativa e dinâmica, bem como utilizado em conjunto com a estratégia BYOD, que os professores pedem para os alunos trazerem os seus dispositivos móveis.

Outra ferramenta utilizada é o Quizlet, que é uma plataforma de estudos disponibilizada para dispositivos móveis quanto e Computadores Pessoais (PC). Segundo TechTudo (2017, p.1), "[...] é possível criar seus próprios flash cards - slides interativos com pergunta e resposta para ajudar na formação de vocabulário -, acessar de outras pessoas, jogar jogos educativos e estudar idiomas online.". O acesso ao serviço é acessado pelo ícone representado no logo do Quizlet, que é disponibilizado na área de aplicativos do browser." (TECHTUDO, 2017, p. 1). A ferramenta Quizlet tem a possibilidade de acessar flash cards prontos, bem como criar os seus próprios. 
Além disso, é possível utilizar os instrumentos de aprender, cards, escrever, avaliar, combinar e gravidade e ter a possiblidade do professor criar jogos em equipe para a fixação de conteúdo, de forma interativa e dinâmica. Há também o recurso de diagramas, que é um estudo voltado para analisar gráficos, imagens e mapas, permitindo adicionar uma imagem que se insere os termos e as definições (TECHTUDO, 2017, p. 1).

\subsection{Materiais e Métodos}

As tecnologias de games e os dispositivos móveis estão presentes no cotidiano de boa parte dos alunos. A partir disso, o intuito foi desenvolver uma dinâmica de jogo que despertasse a motivação dos alunos e que fosse capaz de ajudar de maneira atrativa e de forma significativa os conteúdos da área de computação trabalhados em sala de aula por intermédio dos dispositivos móveis. Na sala de aula a proponente destaca alguma das estratégias que são utilizadas e foram abordadas na seção 2.1 (sala de aula invertida, gamificação, aprendizagem baseada em problemas e aprendizagem baseada em projetos). Essas estratégias são utilizadas em conjunto com as ferramentas abordadas na seção 2.2 (mentimeter e quizlet). A atividade pedagógica teve como sujeitos participantes com uma amostragem de 74 sujeitos participantes de cursos de graduação de computação.

Os 74 sujeitos participantes da amostragem foram orientados previamente pelo professor regente da disciplina, que aplicou o questionário de avaliação a acessarem o link disponibilizado pelo google docs, que contém o Termo de Consentimento Livre e Esclarecido (TCLE). Após a leitura e compreensão do TCLE, caso fosse de interesse e com o consentimento livro e esclarecido o sujeito participante da pesquisa tinha acesso ao questionário de avalição. Pela Figura 1a é possível comprovar que a pesquisa foi realizada com 74 sujeitos participantes da pesquisa de metodologias ativas na arte de ensinar, sendo que $4 \%(n=3)$ dos participantes tem idade superior a 37 anos, $4 \%(n=3)$ possuem entre 32 e 36 anos, 4\% ( $n=3)$ possuem entre 27 e 31 anos, 33\% ( $n=24)$ possuem entre 22 e 26 anos e $55 \%$ ( $n=41)$ possuem entre 17 e 21 anos.

\subsection{Análise e Discussões}

Ao se avaliar a aceitação da metodologia ativa pelos discentes foi possível que $95 \%$ $(n=70)$ dos sujeitos participantes da pesquisa aprovam a utilização das metodologias ativas em sala de aula (Figura 1b), permitindo que se aprenda o conteúdo de forma significativa. Isso pode ser confirmado com 58\% $(n=43)$ dos alunos afirmando que aprovam o uso do método em sala de forma integral, 37\% $(n=27)$ aprovam a utilização do método de forma parcial, somente $5 \%(n=4)$ responderam que desaprovam de forma parcial e $0 \%(n=0)$ respondeu que desaprova totalmente. Portanto, pode-se observar 
que o instrumento pode ser utilizado em ambientes que se deseja instigar e motivar os alunos a aprender e integrar o conhecimento aprendido, resultando em uma aprendizagem de maior qualidade que é a do tipo significativa.
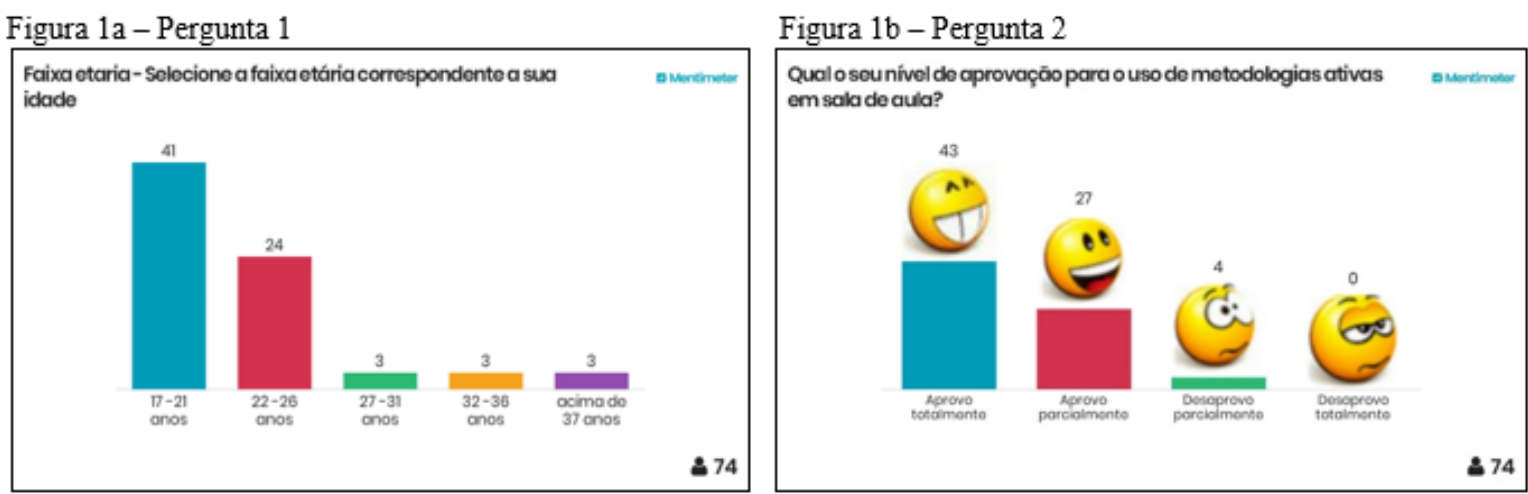

Relacionado aos alunos não sentirem falta da metodologia tradicional para complementar o tema abordado em sala de aula, 92\% $(n=68)$ estão satisfeitos com 0 uso das novas metodologias. Isso pode ser comprovado pela Figura $2 a$ que traz $51 \%$ $(n=38)$ totalmente satisfeitos, $41 \%(n=30)$ que ficam satisfeitos com o uso de forma parcial, $8 \%(n=6)$ que ficaram parcialmente insatisfeitos e $0 \%(n=0)$ que ficaram insatisfeitos totalmente. Pode-se perceber tanto pela literatura quanto pelos números apresentados na pesquisa que a metodologia tradicional padece uma crítica por não conseguir ser apto de contemplar aos interesses e necessidades da sociedade atual. Entretanto, não existe um novo modelo implementado de forma eficaz a ser seguido. Dessa forma, alunos do ensino superior continuam sendo ensinados na escola tradicional e vistos muitas vezes como crianças, reduzindo o encorajamento do pensamento crítico e sustentando a aceitação de respostas prontas oferecidas pelos professores (docentes) aos alunos (discentes).

Referente à participação durante a atividade proposta $96 \%(n=71)$ dos alunos afirmam que a metodologia estimula a participação de todos, pois na aula expositiva dialogada a timidez é um dos fatores que dificultam uma maior interação, fazendo com que o aluno na grande maioria das vezes não participe durante uma discussão e não exponha 0 conteúdo pesquisado. Nas metodologias ativas esse é um aspecto positivo em virtude de outras habilidades serem trabalhadas em conjunto com o tema abordado, bem como é estimulado o trabalho em equipe. Santana, Cunha e Soares (2012) colocam que "Cooperação, desenvolvimento da imaginação, avaliação, normatividade e respeito profissional além de aprender a ouvir, receber e assimilar críticas são exemplos de habilidades desenvolvidas em atividades em grupo nas metodologias ativas.". Isso pode ser comprovado pela Figura $2 \mathrm{~b}$ que traz $66 \%(\mathrm{n}=49)$ dos sujeitos participantes responderam que ficam totalmente motivados com o uso das novas metodologias, $30 \%$ $(n=22)$ que ficam satisfeitos com o uso de forma parcial, $4 \% \quad(n=3)$ que ficaram parcialmente desmotivados e $0 \%(n=0)$ não teve que ficasse desmotivado de fato. 
Figura $2 \mathrm{a}-$ Pergunta 3

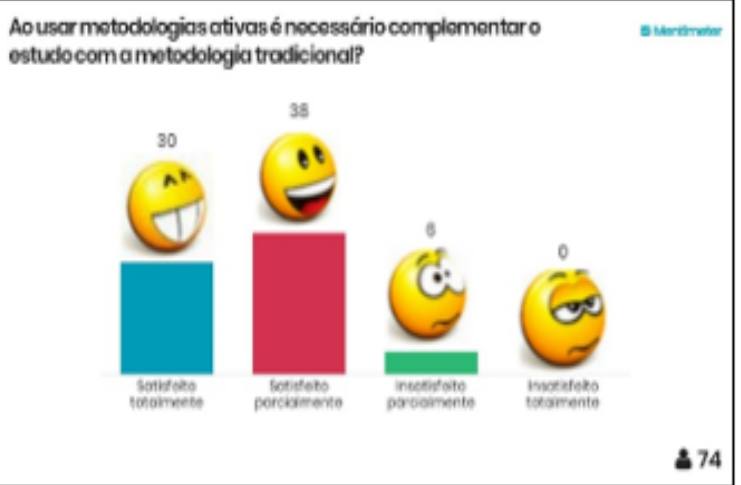

Figura $2 b-$ Pergunta 4

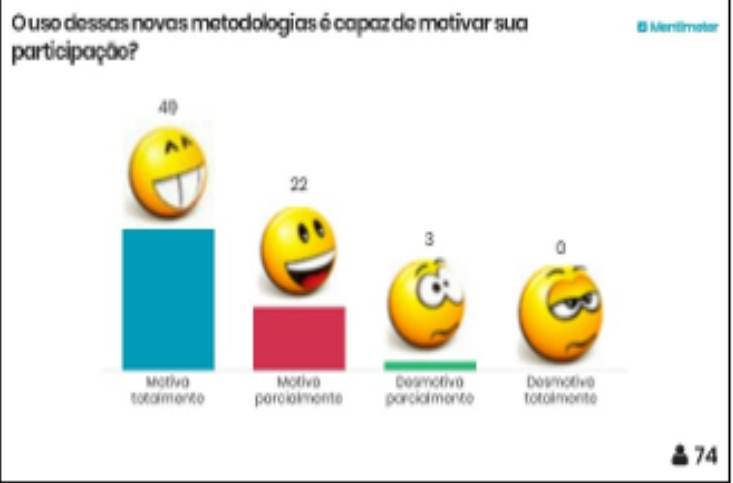

Relacionado a ter ficado com dúvidas após a discussão, 96\% $(n=70)$ sentiram interesse em estudar mais o assunto discutido. Os sujeitos participantes da pesquisa costumam procurar aprofundamentos dos temas dentro dos próprios instrumentos disponibilizados, fazendo uma análise do que é mais relevante estudar e terem um melhor entendimento referente ao tema estudado. Na Figura 3a é possível comprovar esse fato, devido que $60 \%(n=44)$ dos sujeitos participantes responderam que ficam totalmente interessados com o uso das novas metodologias, $36 \%(n=26)$ que tem interesse com o uso de forma parcial, $4 \%(n=3)$ que ficou parcialmente insatisfeito e $0 \%(n=0)$ não teve que ficasse desmotivado de fato com o uso de metodologias ativas.

Enquanto a possibilidade de se utilizar de forma mais ativa metodologias que estimulem uma maior interação e engajamento dos alunos em algumas aulas 99\% $(n=71)$ dos sujeitos participantes da amostragem acharam que seria vantajosa a mudança. Sendo esse resultado comprovado pela Figura 3b, sendo 49\% $(n=21)$ dos sujeitos participantes da amostragem viram o uso das nas novas metodologias melhor que o uso das uso das metodologias tradicionais, $47 \%(n=34)$ viram como de forma complementar, $3 \%(n=2)$ viu de forma equivalente e $1 \%(n=1)$ dos sujeitos participantes da pesquisa viu como pior o uso das novas metodologias.

Figura 3a - Pergunta 5

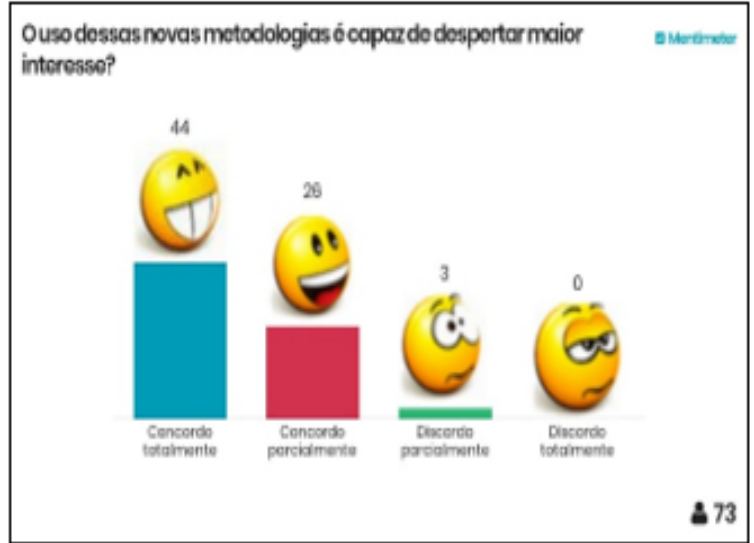

Pergunta $3 b-$ Pergunta 6

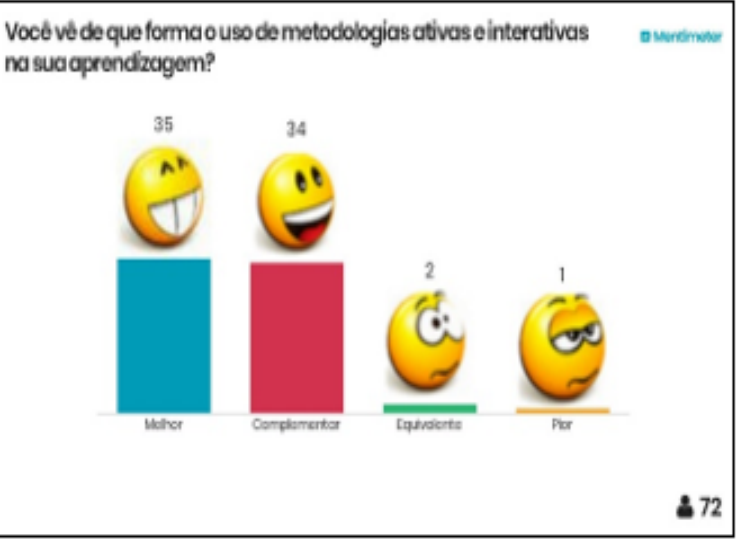




\section{Considerações Finais}

As últimas décadas foram avaliadas com indicadores usando o uso da avaliação formativa e compartimentada em Educação Superior de forma considerável para considerando a qualidade do ensino-aprendizagem; bem como pode-se dizer que as competências relacionadas às capacidades cognitivas e a aprendizagem a longo prazo da vida despertam um maior interesse, motivação e participação do aluno. Além disso, elas promovem uma experiência de ensino-aprendizado, desenvolvendo um maior senso de direitos, deveres e responsabilidades; a correção dos erros; possibilidade de uma maior autonomia dos alunos se comunicarem, desenvolvendo a capacidade de autocrítica e o reconhecimento acadêmico.

Os instrumentos utilizados abordados na pesquisa tiveram impactos positivos, pode-se citar em primeiro lugar um aprofundamento dos alunos na aula, constatando-se uma participação mais ativa nas atividades em sala de aula ao se fazer uso de metodologias ativas, quando comparadas as metodologias tradicionais. Também foi possível perceber que os alunos ficam mais motivados e estimulados pelo tema estudado.

Ressaltasse que foi observado um impacto considerável quanto a participação dos alunos, quando comparadas as duas metodologias. Os alunos passam a comentar suas experiências frente à turma, tecendo comentários pertinentes ao tema estudado em aula, acarretando em uma maior produtividade com as dinâmicas utilizadas em sala de aula. Dessa forma, foi possível perceber um entendimento do pensamento computacional mais sagaz ao se comparar com as metodologias aplicadas de forma tradicional. Pois, ao se fazer uso das novas metodologias os alunos buscam 0 aprendizado de cada item, por si só, refletindo com as dinâmicas aplicadas. Por fim, foi possível verificar que as metodologias ativas auxiliam no processo de ensinoaprendizagem.

\section{Referências}

ALMEIDA, lasmim Andrade. Uma proposta para o ensino da disciplina instrumentação eletrônica no curso de graduação em engenharia elétrica da universidade federal do maranhão. 2017.

BARBOSA, Eduardo Fernandes; MOURA, Dácio Guimarães. Metodologias ativas de aprendizagem na educação profissional e tecnológica. B. Tec. Senac, Rio de Janeiro, v. 39, n. 392, p. 48-6748, 2013.

CHRISTIANINI, Shelley Navari; DE GRANDE, Fernando Chade; AMÉRICO, Marcos. Desenvolvimento de sistemas gamificados com foco no edutretenimento e no jogador: uma análise dos arquétipos de Bartle e Marczewski. Revista Ibero-Americana de Estudos em Educação, v. 11, n. 25, 2016 
DELPHINO, Fátima Beatriz de Benedictis. O papel das aprendizagens ativas na era da sociedade da informação. Anais do Fórum de Metodologias Ativas (MetA). Pontifícia Universidade Católica de São Paulo (PUCSP), Perdizes, São Paulo, 2015. Disponível em: http://www.pucsp.br/rendo-saberes-da-universidade. Acesso em: 01 de abr. de 2018.

INFOGEEKIE. 5 recursos digitais poderosos para você integrar às suas aulas em 2018. 2017. Disponível em: . Acesso em: 7 abr. 2018.

KLAFKE, Guilherme Forma. Uso de aplicativos para avaliações, feedbacks e exercícios. Anais do Fórum de Metodologias Ativas (MetA). Pontifícia Universidade Católica de São Paulo (PUCSP), Perdizes, São Paulo, 2015. Disponível em: $<$ http://www.pucsp.br/reunindo-saberes-da-universidade>. Acesso em: 01 de abr. de 2018

KRAJCIK, J.; BLUMENFELD, P. Project-Based Learning. In: Sawyer, R. The Cambridge Handbook of the Learning Sciences. $1^{\underline{a}}$ ed. New York: Cambridge University Press. 2006.

LUCIO, Carlos Federico. Uso de aplicativos para avaliações, feedbacks e exercícios. Anais do Fórum de Metodologias Ativas (MetA). Pontifícia Universidade Católica de São Paulo (PUCSP), Perdizes, São Paulo, 2015. Disponível em: $<$ http://www.pucsp.br/reunindo-saberes-da-universidade>. Acesso em: 01 de abr. de 2018.

OECD. Measuring Innovation in Education: A New Perspective, Educational Research and Innovation, OECD Publishing, 2014.

PORVIR. Traga seu próprio dispositivo (BYOD). 2015. Disponível em: . Acesso em: 7 abr. 2018.

SANTANA, Alencar; CUNHA, Lopes; SOARES, Alves. Avaliação discente sobre a metodologia de ensino baseado em problemas na disciplina de Farmacologia. Revista Brasileira de Farmácia (RBF), v. 93, n. 3, p. 337-340, 2012.

SANTOS, J.; PASSOS, J. Análise dos benefícios da aprendizagem baseada em problemas (ABP) no desenvolvimento de projetos práticos no curso de engenharia da Universidade Virtual do Estado de São Paulo (UNIVESP). In: Problem Based Learning International Conference. São Paulo. 2016.

SOUZA, Ricardo André Cavalcante de. Inovação em Projetos de Software aplicada a Soluções Educacionais. VI Congresso Brasileiro de Informática na Educação (CBIE 2017) Anais do XXIII Workshop de Informática na Escola (WIE 2017), 2017.

TECHTUDO. Quizlet é uma plataforma de estudos disponível para PC e celulares. 2017. Disponível em: . Acesso em: 7 abr. 2018. 